nano-printing

\title{
Nanoscale plasmonic printing
}

Soon Hock Ng and Saulius Juodkazis

Optical Sciences Centre, School of Science, Swinburne University of Technology,

Hawthorn, Vic 3122, Australia

(Dated: 19 February 2022)

Nanoscale structuring/printing is of interest for range of applications in 3D subtractive and additive manufacturing $\left(3 \mathrm{D}^{ \pm}\right)$. Basic principles of light field enhancement and control at the nanoscale are overviewed in this section/chapter for bulk, surface, and localised plasmons (1D, 2D, and 3D localisation, respectively). All these plasmons are resonant phenomena which have common Lorentzian spectral lineshape which relates refractive and absorption properties as well as defining the phase of transmitted and scattered light. Localisation of light at the nanoscale creates the possibility of modification with matching resolution. Harnessing this light enhancement can be demonstrated as a "nano-pen" for direct write nanolithography.

Keywords: plasmonics, nanoscale, ablation, direct laser write

\section{CONTENTS}

$\begin{array}{ll}\text { I. Why plasmonic? } & 1\end{array}$

II. Surface plasmon polariton (SPP) 3

III. Photo-generated plasma 4

A. Permittivity defined by the electron density $N_{e}$ and collision rate $\nu \quad 4$

B. Dielectric-to-Metal transition: permittivity analysis 5

C. Energy deposition at the ENZ-conditions $(\varepsilon \rightarrow 0) \quad 7$

$\begin{array}{ll}\text { D. Electron photo-generation } & 9\end{array}$

IV. Nanoparticles in high intensity laser field 11

V. Nanolithography by direct write with optical near-field 15

$\begin{array}{ll}\text { VI. Localisation of energy deposition } & 17\end{array}$

$\begin{array}{ll}\text { VII. Conclusions and outlook } & 17\end{array}$

$\begin{array}{ll}\text { Acknowledgments } & 18\end{array}$

\section{WHY PLASMONIC?}

Light-matter interaction is determined by the the relative permittivity (dielectric function) of a material $\left.\varepsilon(\omega)\right|_{t_{0}}$ at the wavelength of light $\lambda$ and at a particular moment in time $t_{0}$ from the material side; $\omega=2 \pi c / \lambda$ with $c$ being speed of light. The light intensity $I(t) \equiv E(t)^{2}$ is defined by its electric field $E$ at that selected moment in time $t_{0}$ and defines how much the light's energy is absorbed, reflected, scattered, and transmitted. All the portions of the absorbed, transmitted and reflected light obeys energy conservation $A+R+T=100 \%$. The real and imaginary parts of the permittivity $\varepsilon \equiv \varepsilon_{1}+i \varepsilon_{2}$ defines the dispersion and absorption related response of a material; permittivity is related to complex refractive index $\sqrt{\varepsilon} \equiv \tilde{n}=(n+i \kappa)$. In such a description, a strongly nonlinear response of a material in the field of a high intensity laser pulse is given by the instantaneous value of $\varepsilon$ (or $\tilde{n}$ ). For a material exposed to a short pulse of high intensity, electrons are generated by 


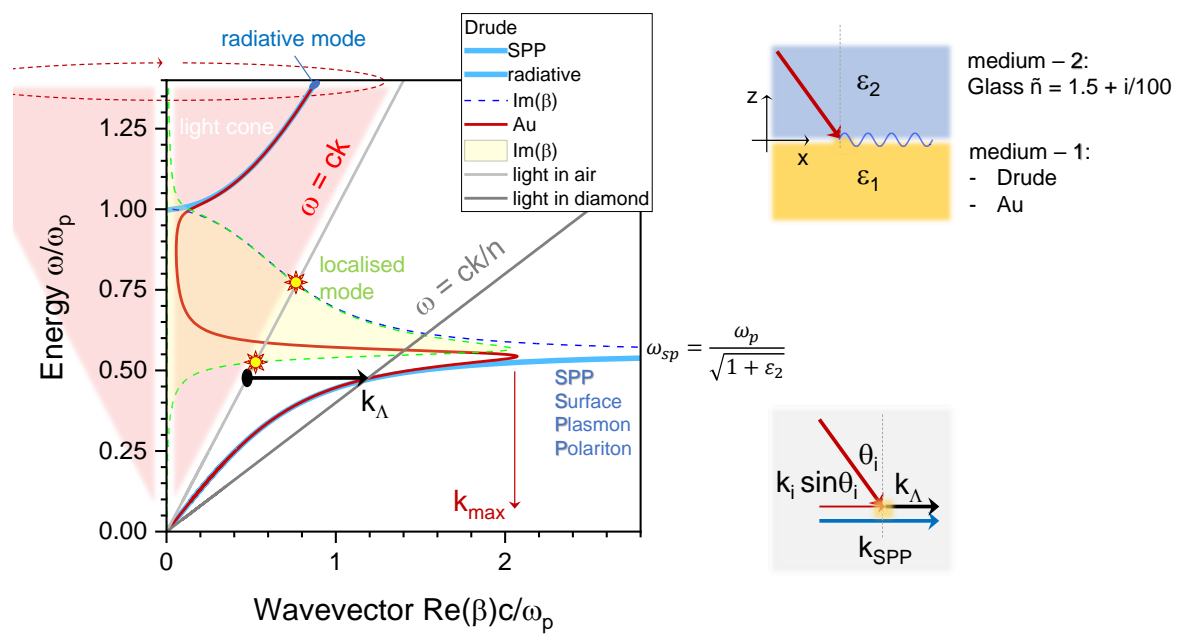

FIG. 1. Dispersion $\omega(k)$ relations for Drude models: lossless and Au at the metal-glass interface; $\omega_{p}$ is the plasma frequency and glass has refractive index of $n=1.5+i / 100$. Phase matching diagram $k_{S P P}=k_{i} \sin \theta_{i}+k_{\Lambda}$ to launch surface plasmon polariton (SPP) with a grating (or prism) introducing $k_{\Lambda} ; k_{i}, \theta_{i}$ are the wavevector of incident light and angle of incidence, respectively (rightbottom inset). Refractive index of a $n=2.5$ dielectric (e.g. a diamond prism) for the light-line $\omega=(c / n) k$ is shown. Dashed lines are $\omega$ vs. $\operatorname{Im}(\beta)$ (non-propagating localised mode), where $\beta$ is the propagation constant or SPP wavevector (Eqn. 1) at the metal-glass interface. An asymptotic frequency of surface plasmon $\omega_{s p}$ for the loss less Drude plasma (no electron damping $\operatorname{Im}\left(\varepsilon_{D}\right)=0$; medium - 1$) ; \varepsilon_{2}$ is permittivity of glass (medium - 2). Insets show diagrams of interaction zone (right-top) with scattering of a dipole nature providing redirection and wave matching.

linear and nonlinear mechanisms of absorption. Light propagation and absorption in such a photo-excited state is described by nonlinear optical methods until this solid-state plasma state transitions into a true ionised plasma state defined by dielectric breakdown at $\varepsilon=0$.

The most interesting and highly dynamic changes to material relevant to the discussion here occurs during transition to plasma and pre-breakdown conditions. In the case of ultrashort sub-1 ps pulses of high intensity, the transition from solid to plasma state can be very fast, occurring over several optical cycles ( $\sim 3 \mathrm{fs}$ ) at the laser wavelength $\lambda \approx 1 \mu \mathrm{m}$. It's no surprise that a complete understanding of complex spatio-temporal phenomena evolving in time and along the light propagation direction inside excited micro-volumes attracts interest and is analysed via increasingly sophisticated experimental techniques.

In the process of defining the scope of this discussion, how it pertains to light-matter interactions within a photo-excited focal volume and transition from a dielectric to a metallic state $1<\varepsilon<0$, we in fact discuss plasma related phenomena occurring on the inner surface dielectric (transparent) host volume. Hence, the word plasmonic is well aligned to such field of research and applications.

As an example, plasmonic printing is where modifications are induced in a material by light localised by a nanoparticle or a sharp tip in close proximity the sample being modified. Such printing can be nanoscale, with sub-wavelength feature sizes below the classical optical resolution limit $\sim 0.6 \lambda / N A$, defined by the numerical aperture $N A$ of the lens used. True nanoscale localisation by this plasmonic prinitng can be achieved when the modified volume is constrained to dimensions smaller than $100 \mathrm{~nm}$ along all axes, thus nanoscale.

In experiments with light-matter interactions, when ultra-short sub-1 ps laser pulses create sub-wavelength and nanoscale patterns (nanoprints) on surfaces and interfaces, the surface waves at those interfaces are discussed. The interfaces can be a natural boundary at sample's surface with its environment (air, gas, liquid, other solid) or can be created inside a transparent host medium at the focal region where photo-excitation creates a highly conductive (plasma) volume via absorption and ionisation. In both cases, the interface be- 
nano-printing

tween materials with different permittivity has to be analysed, accounting for fast changing permittivities. Such analysis is presented next, considering explicitly the interface between two mediums with complex permittivities.

\section{SURFACE PLASMON POLARITON (SPP)}

As a surface wave, light bound to the collective oscillation of surface charge, the surface plasmon polariton (SPP) can exist at the interface of metal and dielectric (medium 1 and 2 in Fig. 1). Dispersion relation $\omega$ vs. wave number $k=2 \pi / \lambda$ is found from the solution of the wave Helmholtz equation. The electrical E-field of the SPP wave has a form $E_{x} e^{i \beta x} e^{-k_{z} z}$, describing propagation along $\mathrm{x}$-direction with a propagation constant $\beta$ (the wavevector):

$$
\beta=k_{0} \sqrt{\frac{\varepsilon_{1} \varepsilon_{2}}{\varepsilon_{1}+\varepsilon_{2}}},
$$

where $k_{0}=2 \pi / \lambda$ is the wavevector of incident light ( $k_{i}$ in Fig. 1). The SPP, an evanescent field, exponentially decays into the surrounding medium 1 and 2 with its own $k_{z}^{(1,2)}$. As the SPP approaches the surface plasmon frequency $\omega_{s p}=\omega_{p} / \sqrt{1+\varepsilon_{2}}$ - where the plasma frequency $\omega_{p}=\sqrt{N_{e} e^{2} /\left(\varepsilon_{0} m\right)}$ (also referenced as bulk plasmon) is defined by electron density $N_{e}$, electorn charge and mass $e, m$, respectively, and permittivity of free space $\varepsilon_{0}$ the permittivity of metal (negative) has the same absolute value as the the dielectric host $\varepsilon_{1}(\omega)+\varepsilon_{2}=0$ and the group velocity of the SPP $v_{g} \rightarrow 0$ (a standing wave).

Figure 1 shows dispersion relations (Eqn. 1) for the metal-glass (2-1) interface where metal (1) is modeled by the Drude model and its adaptation to the $\mathrm{Au}^{1}$. Dielectric medium (2) is glass-like with permittivity $\varepsilon_{2} \equiv \tilde{n}=(1.5+i / 100)^{2}$. The Drude model is useful to understand the underlying physics of SPP dispersion. The plasma of a free electron gas in the fixed ion background that provides a restoring action for the oscillation is described by the permittivity separated into real and imaginary parts $\left(\varepsilon_{1}\right.$ medium in Fig. 1$)$ :

$$
\varepsilon(\omega)=1-\frac{\omega_{p}^{2}}{\omega^{2}+i \gamma \omega}=\left(1-\frac{\omega_{p}^{2} \tau^{2}}{1+\omega^{2} \tau^{2}}\right)+i \times \frac{\omega_{p}^{2} \tau}{\omega\left(1+\omega^{2} \tau^{2}\right)},
$$

where $\tau=1 / \gamma$ is the lifetime of the electron plasma oscillation defined by its collision frequency $\gamma$. At low frequencies $\omega<\omega_{p}$, metallic plasma behaves like a metal (are reflective). Closer to $\omega_{p}$, the product $\omega \tau \gg 1$ and the damping of oscillations is negligible and $\varepsilon(\omega)$ (Eqn. 2) is dominated by the real part defined by the undamped free electron plasma $\varepsilon(\omega)=1-\omega_{p}^{2} / \omega^{2}$. This ideal condition of the Drude model $\varepsilon_{D}(\omega)$ is plotted in Fig. 1. It has both the SPP mode for the lower frequency branch discussed above and the high-frequency $\omega / \omega_{p}>1$ where plasma is optically transparent and has dispersion of $\omega^{2}=\omega_{p}^{2}+k^{2} c^{2}$ for transverse traveling waves with group velocity $v_{g}=d \omega / d k<c$. This branch of dispersion is less relevant to the discussion here were radiative E-fields escape the light-matter interaction volume. The radiative modes are confined inside the light cone around the Energy-axis with boundary if the light line $\omega=c k$ (shaded region in Fig. 1). This light cone with $x$ as a radial coordinate also defines all the possible projections of the wavevector $k$ for transverse waves at a specific wavelength (or $\omega$ ). Such presentation is useful for the discussion of the phase matching conditions necessary to launch the surface bound transverse TM-mode SPP wave. Phase matching is provided by scattered light along the direction of SPP wave. Scattering provided by roughness, which can be actual surface roughness, caused by an unevenness of permitivity, or can be dipole scattering of a nanoscale localised absorber, e.g., a nanopartcle. For a controlled launch of a SPP, such phase matching is provided by a wavevector of a grating or under total internal reflection at a specific angle of incidence $\theta_{i}$. Regardless of the phase matching mechanism, it is marked as $k_{\Lambda}$. A diamond prism can provide the required wavevector deficit from the light cone in air, to the SPP mode at the intersection point with the light-line, in a medium of refractive index $n=2.5$ (diamond); see Fig. 1. 
nano-printing

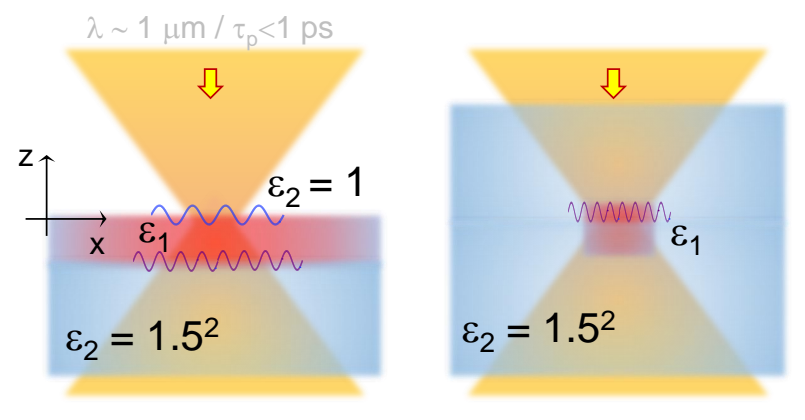

FIG. 2. Geometry of interfaces between photo-excited material transitioning via a dielectric-metal (Die-Met-red region) state of permittivity with focal spot placed on the surface or inside a transparent medium (permittivity $\varepsilon_{2}=1.5^{2}$ of a glass-like host-blue region).

Plasmonic (Olympic) metals Au, Ag, Cu can be modeled with the Drude model at lower frequencies when interband electronic transitions don't place (up to $1 \mathrm{eV}$ energy). This is called the Drude-Sommerfeld free electron model. For gold, the best fit of experimentally measured permittivity to Eqn. 2 is achieved with $E=\hbar \omega_{p}=8.5 \mathrm{eV}$ and $\tau=14 \mathrm{fs}^{1}$. These values are close to other reported data and were used for the $\mathrm{Au}$ dispersion plot shown in Fig. 1. The time constant $\tau$ is a fit parameter which reflects cumulative charge scattering interactions. With this Drude-Sommerfeld model of Au permitivity, the dispersion dependence of a SPP mode on an Au-glass interface has a strong overlap with the lossless Drude model, however, there is a distinct maximum value of the wavevector $k_{\max }=2 \pi / \operatorname{Re}(\beta)$. $k_{\max }$ is proportional to $\tau$. The $k_{\max }$ for Au with $\hbar \omega_{p}=8.5 \mathrm{eV}^{1}$ (Fig. 1) corresponds to smallest wavelength of SPP of $\lambda_{s p}=70.6 \mathrm{~nm}$. The value of $2 \pi / \operatorname{Re}(\beta)$ is usually used to define the period of laser imprinted ripples also called light induced periodic surface structures (LIPSS), even though they can be formed inside materials or sub-surface as discussed next.

\section{PHOTO-GENERATED PLASMA}

\section{A. Permittivity defined by the electron density $N_{e}$ and collision rate $\nu$}

Conditions required to launch a SPP at a metal (or a plasma) - dielectric interface are described above (Sec. II). They are dictated by the electron density $N_{e}$ which defines the plasma frequency $\omega_{p}$ and relaxation time presented by an effective $\tau$. Under irradiation of a high-intensity 1-100 $\mathrm{TW} / \mathrm{cm}^{2}$ ultra-short laser pulse, a dielectric or semiconductor can be converted into a metallic state with $0<\varepsilon<1$ and dielectric breakdown can be induced at the focal spot defined by $\varepsilon=0$. This fast material transformation during sub- 1 ps pulse can be considered as a material of changing state (dielectric-metal - Die-Met ${ }^{2}$ ). Once an $\varepsilon<1$ region focal volume is created, a SPP at the interface with a dielectric host can be launched. For the surface of a dielectric or semiconductor in the focal region of a high intensity pulse, two interfaces have to be considered, one at the internal interface between the host and surface bound Die-Met, as well as Die-Met with ambient air (Fig. 2).

In a similar way as the Drude model is modified to define permitivity of Au with two parameters $\omega_{p}$ and $\tau$, it can be used for semiconductors and dielectrics which have a large real part of permittivity (refractive index), which decreases under photo-generation of electrons ${ }^{2}$ :

$$
\varepsilon=\left[\left(\varepsilon_{i}-1\right) \frac{N_{e}}{N_{a}}\right]+\left[1-\frac{N_{e}}{N_{c r}(1+i \nu / \omega)}\right]
$$


where $N_{a}=\rho N_{A} / M$ is the atomic density expressed via mass density, Avogadro number $N_{A}$ and the molar mass $M, N_{c r}=\varepsilon_{0} \omega^{2} m / e^{2}$ is the critical number density and $\nu$ is the effective collision frequency (its dependence on $N_{e}$ and temperature of electrons $T_{e}$ is discussed below). The $N_{a}$ is a source of electrons upon optical excitation. Equation 3 is derived by making use of identity $\omega_{p}^{2} / \omega^{2}=N_{e} / N_{c r}{ }^{3}$. The first term in brackets [...] describes the reduction of unperturbed permittivity due to induced polarisation of the material while the second [...] term is Drude like due to electronic part of the permittivity. The $N_{c r}$ defines electron density at which the material becomes reflective, i.e., the plasma frequency equals the frequency of light. For example, for the $\lambda=1030 \mathrm{~nm}$ wavelength $N_{c r}=1.05 \times 10^{21} \mathrm{~cm}^{-3}$.

Collision rates ${ }^{3}$. The elastic momentum exchange rate or collision rate $\nu$ of electrons is effectively a constant due to collisions with the lattice (phonons), neutrals, electrons, and ions ${ }^{3}$. The following estimates are obtained for numerical modeling and follows ref. ${ }^{3}$. The collision rate is dependent on concentration and temperature increasing in intensity as both do. The quasi-neutrality between electrons and ions $N_{e}=N_{i}$ is maintained $^{3}$. When $N_{e} \ll N_{a}$, electron-phonon interactions dominate and at room temperature $\nu_{e-p h} \approx$ $k_{B} T / \hbar=3.79 \times 10^{13} \mathrm{~s}^{-13}$, here $k_{B}$ is the Boltzmann's constant. The electron-ion Coulomb collisions increase with density towards the upper physical limit $\nu_{e-i}^{\max } \approx v_{e} / d \approx 10^{16} \mathrm{~s}^{-1}$, where $v_{e}$ is the electron velocity and $d$ is the interatomic distance $d=1 / \sqrt[3]{\frac{3 N_{a}}{4 \pi}}$. The Coulomb collisions increase with laser pulse intensity and dominate at the ablation threshold when first ionization occurs, i.e., $N_{e} \approx N_{a}$. The maximum rate of collisions $v_{e-i}^{\max }=v_{e}^{\max } / d$, where the velocity $v_{e}^{\max }=\sqrt{2\left(J_{i}+\epsilon_{B}\right) / m_{e}}, J_{i}$ is the ionization potential and $\epsilon_{B}$ is the binding energy (the enthalpy of formation, the heat of sublimation, or latent heat ${ }^{4}$ - for example for $\mathrm{Si} \epsilon_{B}=4.62 \mathrm{eV} /$ atom). The electron energy at the ablation threshold is equal to the sum $J_{i}+\epsilon_{B}$. This amount of electron energy is sufficient to break bonds inside the solid.

With increasing laser intensity and temperature, the collision rate decreases as in an ideal plasma $v_{e-i} \propto N_{e} / T_{e}^{3 / 2}$. The collision rate $\nu_{e f f}=N_{e} \sigma_{\Sigma} v_{e}$ is defined by the sum of cross sections $\sigma_{\Sigma}$ of the contributing processes in momentum transfer from electrons to ions. The description above justifies definition of collision rate as:

$$
\nu\left(N_{e}\right)=\nu_{e-p h}^{\min }+\nu_{e-i}^{\max } \frac{N_{e}}{N_{a}} .
$$

$\mathrm{SiO}_{2}$ Case. Next, estimates are made for $\mathrm{SiO}_{2}: d \approx 0.575 \mathrm{~nm}$ (with mass density $\rho=$ $2.2 \mathrm{~g} / \mathrm{cm}^{3}$, molar mass $\left.M=28+2 \times 16=60 \mathrm{~g} / \mathrm{mol}\right), \epsilon_{B}=3.7 \mathrm{eV} /$ atom $^{5}$ with the larger ionisation potential of oxygen $J_{i}=13.6 \mathrm{eV}$ (for Si $J_{i}=8.15 \mathrm{eV}$ ) used for the estimate of $\nu_{e-i}^{\max }=4.29 \times 10^{15} \mathrm{~s}^{-1}$ with velocity $v_{e}^{\max }=2.47 \times 10^{6} \mathrm{~m} / \mathrm{s}$. Evolution of permittivity of $\mathrm{SiO}_{2}$ under increasing ionisation during a high intensity pulse is calculated with Eqn. 2 using electron density dependence of collision rate $\nu\left(N_{e}\right)$ (Eqn. 4) and is plotted in Fig. 3.

\section{B. Dielectric-to-Metal transition: permittivity analysis}

Figure 3(a) illustrates the change in permittivity $\varepsilon$ from a dielectric to a metallic plasma state using collision rate estimates provided above. This qualitative picture of light-matter interaction shows the region where the interface can be formed supporting the SPP mode between plasma $\operatorname{Re}(\varepsilon)<1$ and surrounding glass (or air), see Fig. 2. For a shorter wavelength, the photo-excited region is partly transparent up to larger electron density due to larger $N_{c r}$. Calculations shown in Fig. 3(a) were carried out to the point of first ionisation of atoms, i.e., the $N_{a}$ of $\mathrm{SiO}_{2}$. At high light intensity, multiply ionised plasma is attain$a^{a b l e}{ }^{6,7}$ and its density and temperature will define the actual collision rate which was only estimated qualitatively for herein. However, the physical picture of collision-rate-controlled permittivity changes is shown here with conditions of SPP creation at the interface between plasma and surroundings. It is instructive prediction of SPP on plasma of critical density $N_{c r}$ on glass $\varepsilon_{2}=(1.5+i / 100)^{2}$ shown in Fig. $3(\mathrm{~b})$. The dispersion plot of energy $\omega$ vs. 
(a)

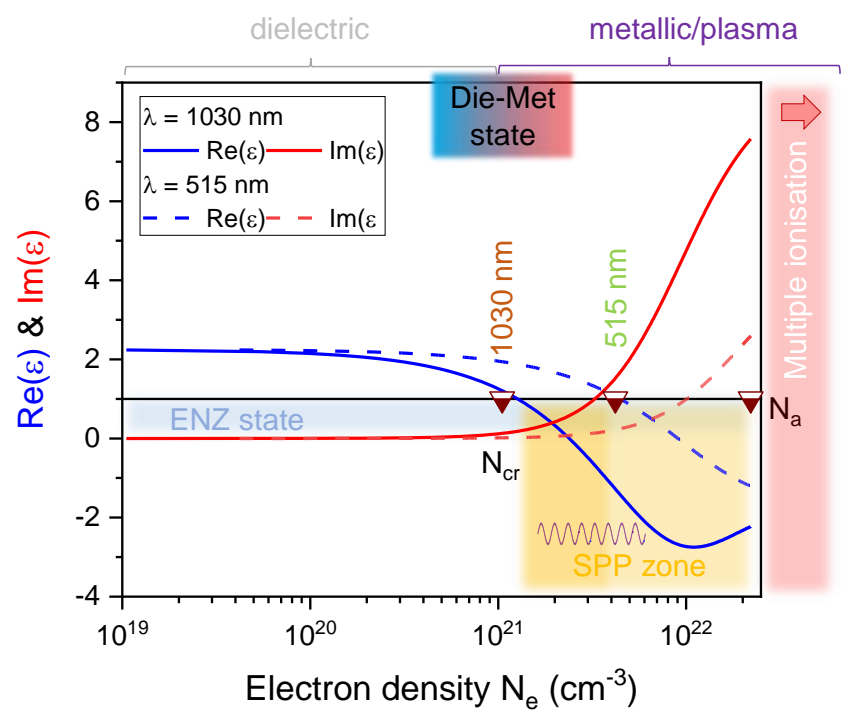

(b)

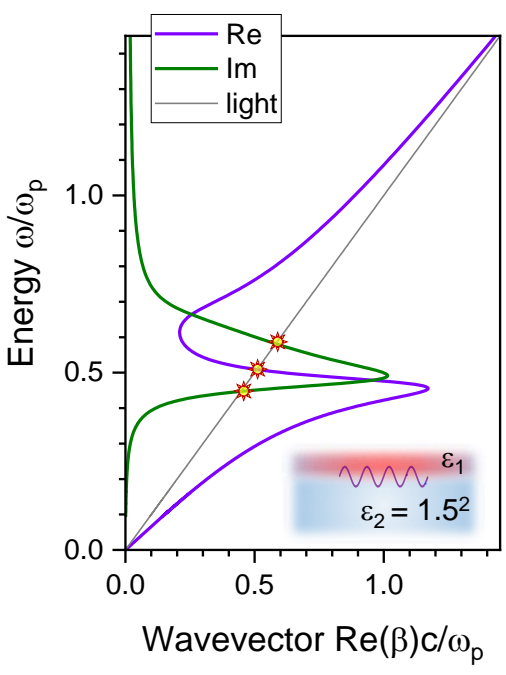

FIG. 3. Dielectric-Metal transition (Die-Met). (a) Silica $\mathrm{SiO}_{2}$ under increasing generation of electrons $N_{e}\left[\mathrm{~cm}^{-3}\right]$; starting refractive index $\sqrt{\varepsilon}=1.5$; the critical density of electrons $N_{c r}$ for two wavelengths $\lambda=1030 \mathrm{~nm}\left(1.05 \times 10^{21} \mathrm{~cm}^{-3}\right)$ and $515 \mathrm{~nm}\left(4.20 \times 10^{21} \mathrm{~cm}^{-3}\right)$ are shown by markers on the $\operatorname{Re}(\varepsilon)=1$ line separating dielectric-metallic state (Die-Met transition). The atomic density of $\mathrm{SiO}_{2} N_{a}=2.21 \times 10^{22} \mathrm{~cm}^{-3}$ is the limit of the curves. Calculations were made with Eqns. 2 and 4 with parameters evaluated in Sec. III. (b) Equation 1 plotted for the epsilon-near-zero (ENZ)material at $N_{c r}$ with $\omega_{p}$ for $\lambda=1030 \mathrm{~nm}$ (Eqn. 3) interfaced with silica $\sqrt{\varepsilon_{1}}=1.5+i / 100$; light line is for air. Markers show intersection locations of the propagating surface wave SPP and localised $\operatorname{Im}(\beta)$ vs. $\omega$.

$\operatorname{Re}(\beta)$ and $\operatorname{Im}(\beta)$ resembles a Lorentzian spectral lineshape of dispersion $D(\omega)$ and absorbance $A(\omega)$, respectively ${ }^{8}$. The dispersion dependence of the surface bound propagating SPP wave (real $\beta$ ) and non-propagating surface bound (imaginary $\beta$ ) are close together in both energy and wavevector space at the intersection with free space light. Such intersection indicates the possibility to couple the free propagating light into those modes and relax the required phase matching conditions described for SPP launched on the lossless Drude plasma (Fig. 1). Such dispersion/absorbtion spectral lineshape resembles a dipole response. During exposure of the dielectric medium to a high-intensity ultra-short laser pulse, the plasma density evolves quickly, creating a constantly changing plasma density. Its temperature and collision rates are all important to the discussion of SPP launch at the interface. The spatial extent of such an interface and energy absorption are also directly linked to the $\varepsilon$ as is analysed next.

Permittivity given by Eqn. 2 defines a state of photo-excited material at which a SPP wave can be launched. Metal-like permittivity $1>\operatorname{Re}(\varepsilon)>0$ created by a laser pulse defines region of interest at this epsilon-near-zero (ENZ) condition which are efficient in light energy absorption. The rate of absorption and its spatial location are important for interface formation required for the SPP. Also, the phase of light upon reflection from the boundary between different refractive index materials change in a step-like manner. The phase of the reflected field $E_{R}$ (incidence from air $n=1$ ) onto material of index $n$ is defined by $E_{R}=-\frac{n-1}{n+1} E_{i n}$, where $E_{i n}$ is the incident E-field and the "-" sign defines the opposite phase or the $\pi$-shift. For $n>1$, there is a $\pi$-shift between the incident and reflected E-fields. Since the real part of refractive index of ENZ $n<1$, there is no phase change between $E_{R}$ and $E_{i n}$. Figure 4 illustrates light incidence on the micro-cylinders 
nano-printing

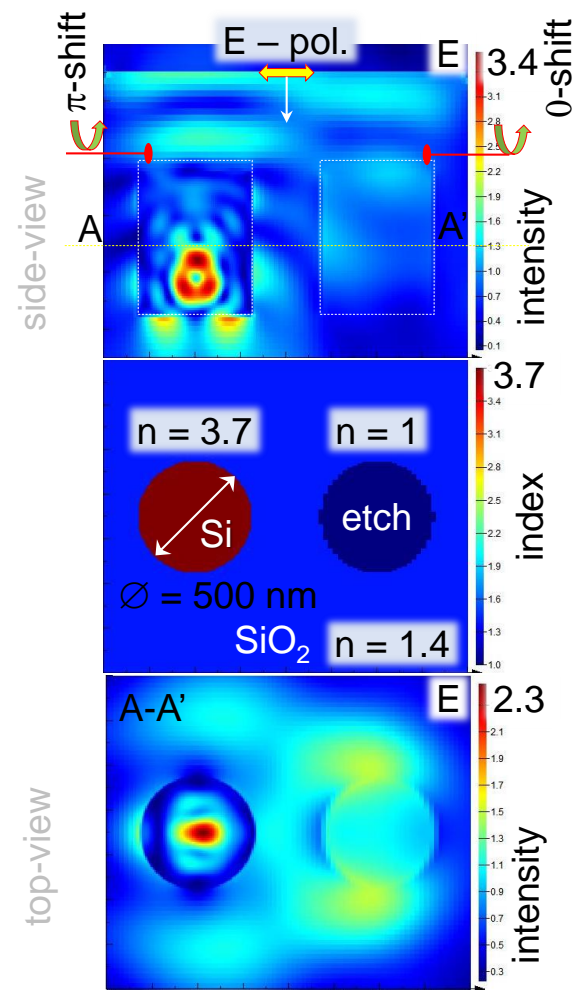

FIG. 4. Numerical modeling of plane wave propagation through micro-cylinders of Si and empty space (etch with refractive index $n=1$ ) inside a glass host $(n=1.4)$; wavelength $\lambda=767 \mathrm{~nm}$. Calculations were made by finite difference time domain (FDTD) simulations (Lumerical). Panels show light field enhancement; incident field is a linearly polarised plane wave with $E=1$. Phase $\pi$-shift occurs upon reflection from a higher refractive index material.

of higher and lower refractive index embedded inside glass. These volumes are similar to those that occur when laser pulses are tightly focused using objective lenses of numerical aperture $N A \approx 1$ in the wavelength range from visible to near IR. Formation of interference fringes at different distances from the front surface of these cylinders are a demonstration of the phase change. This illustration shows that phase changes around the interfaces which support SPP modes can alter light intensity distribution, intensity causing different absorption and plasma density.

\section{Energy deposition at the ENZ-conditions $(\varepsilon \rightarrow 0)$}

This section describes how a material is changed via permittivity evolution with larger electron density $N_{e}$ and simultaneously changing collision rate $\nu$. The conditions discussed are at high electron densities around the critical plasma density at the laser wavelength $0.1<\frac{N_{e}}{N_{c r}}<10$. At around $N_{c r}$, SPP conditions can be created for the interface of excited plasma in contact with a dielectric host (Fig. 3(b)).

Surfaces discussed in plasmonic nanoprinting look like the ablated Si shown in Fig 5 with different period of semi-regular patterns of ripples-light induced periodic surface structures (LIPSS). Ripples can be formed on the surface or inside the volume of dielectrics or semiconductors. Small sub-wavelength ripples on the surface and especially inside the bulk are particular for exposure by high intensity ultra-short laser pulses. Surface patterns are formed by ablation - Latin word for removal - which means that material has enough deposited/absorbed energy to loose solid-state material cohesion (overcome the binding en- 


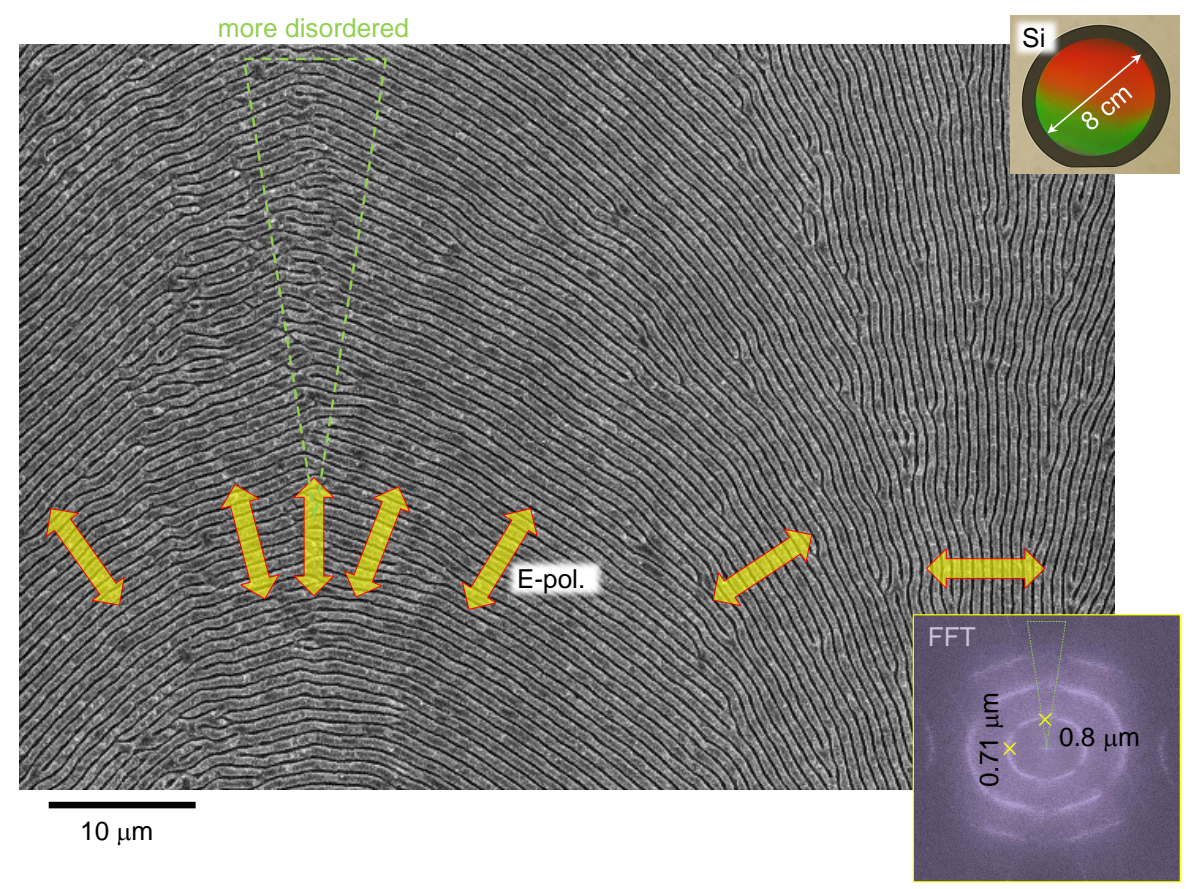

FIG. 5. SEM image of large area nanoprinting of ripples on Si by ultra-short laser pulses: wavelength $\lambda=1030 \mathrm{~nm}$, repetition rate $f=100 \mathrm{kHz}$, overlap of 10 pulses per $1 \mu \mathrm{m}$, scan speed of $v_{s}=0.1 \mathrm{~mm} / \mathrm{s}$, focusing with numerical aperture $N A=0.26$, pulse energy $E_{p}=10 \mu \mathrm{J}$, polarisation rotation $2^{\circ}$ per micrometer of travel. Laser beam was focused with an added cylindrical lens of $f=80 \mathrm{~mm}$ defining the ablation area of $\sim 400 \times 5 \mu \mathrm{m}^{2}$ on the sample (elongated along polarisation). Photo inset is for linear scan without polarisation rotation ${ }^{9}$.

ergy $\epsilon_{B} \sim 5 \mathrm{eV} /$ atom) and to be ionised with ionisation energies $J_{i}>10 \mathrm{eV} /$ atom. Exciting solid sate matter and exceeding energy deposition required for each phase transition - from melting, evaporation $\left(\sim \epsilon_{B}\right)$, to ionisation $\left(J_{i}\right)$, and plasma - within a sub-1 ps pulse is a very fast process since a typical lattice vibrations are on the order of few picoseconds and don't mediate energy deposition by sub-1 ps pulses. The energy deposition by absorbance $A$ is linked to the reflectance $R$ in the overall energy balance $A+R+T=100 \%$ where $T$ is the transmittance. How much light is absorbed and reflected is all defined by the permittivity $\varepsilon$ of the material. As discussed above, $\varepsilon$ changes during a laser pulse since electrons are fast in following the E-field of the incident light.

For light incident from air $(n=1)$ onto a material with refractive index $(n+i \kappa)$, the Fresnel coefficients defines $R$ at angle of incidence $\theta_{i}$ and for the normal incidence $\theta_{i}=0$ it is $R=\frac{(n-1)^{2}+\kappa^{2}}{(n+1)^{2}+\kappa^{2}}$. For insignificant transmission $T \rightarrow 0$ in an absorbing medium (optically thick), $A=1-R$ and is $A=\frac{4 \kappa^{2}}{(n+1)^{2}+\kappa^{2}}$. From Eqn. 3 we can calculate which portion of light is absorbed at different electron densities leading to the ENZ conditions. If the starting material has $n=1.5$ (e.g., silica glass), at $N_{c r}$ for $\lambda=1030 \mathrm{~nm}$ one finds $\sqrt{\varepsilon}=$ $n+i \kappa=0.34+i \times 0.19$. Thus $A=73.8 \%$, which is a substantial energy deposited to the target with the depth of absorption (skin depth for the intensity $I=|E|^{2}$ ) defined as $l_{a b}=\frac{c}{2 \omega \kappa}=\frac{\lambda}{4 \pi \kappa}=427 \mathrm{~nm}$. At $N_{e}=N_{c r} / 2$, the most efficient energy deposition take place, with $A=97.5 \%$. At larger larger plasma density $N_{e}=2 N_{c r}, \tilde{n}=0.25+i \times 0.91, A=41.8 \%$ and deposition depth $l_{a b}=90 \mathrm{~nm}(10 \%$ of $\lambda$ ). Shallow energy deposition is favorable for formation of an interface supporting the SPP mode.

When the plasma frequency approaches that of the collision rate $\omega_{p} \approx \nu$, optical constants 
nano-printing

of plasma become ${ }^{5}$ :

$$
\operatorname{Re}(\varepsilon) \approx \frac{\omega^{2}}{\omega_{p}^{2}} \sim \frac{N_{c r}}{N_{e}} ; \quad \operatorname{Im}(\varepsilon) \approx \frac{\omega_{p}}{\omega}\left(1+\frac{\omega^{2}}{\omega_{p}^{2}}\right)^{-1} ; \quad n \approx \kappa=\sqrt{\operatorname{Im}(\varepsilon) / 2}=\sqrt{\frac{\omega_{p}}{2 \omega}},
$$

i.e., $n \approx \kappa$ as in the example above or glass at electronic density of $2 \times N_{c r}$ with metallic state of $n<1$.

Ablation threshold fluence for dielectrics $F_{t h}\left[\mathrm{~J} / \mathrm{cm}^{2}\right]$ is predicted from considering that the pulse energy is absorbed by electrons in the skin depth $l_{a b}$ and is in excess of the bonding energy and ionisation potential $\left(\epsilon_{B}+J_{i}\right)^{5}$ :

$$
F_{t h}^{D i e}=I_{t h} t_{p}=\frac{3}{4}\left(\epsilon_{B}+J_{i}\right) \frac{l_{a b} N_{e}}{A},
$$

where $t_{p}$ is the pulse duration and $I_{t h}\left[\mathrm{~W} / \mathrm{cm}^{2}\right]$ is the pulse intensity (irradiance). Considering the upper bound estimate with $J_{i}=13.6 \mathrm{eV} /$ atom for oxygen and full ionisation of the material $\left(N_{e}=N_{a}\right)$, one finds that for $A$ and $l_{a b}$ at $N_{c r}, F_{t h}^{D i e}=2.65 \mathrm{~J} / \mathrm{cm}^{2}$ for silica. It is very close to the experimentally determined value of $2 \mathrm{~J} / \mathrm{cm}^{2}$. This is a reasonable estimate since $A$ and $l_{a b}$ are strongly inversely dependent on $N_{e}$ and the experimentally determined threshold fluence is only obtained at $1.1 N_{c r}$.

\section{Electron photo-generation}

The peak intensity of an ultra-short laser pulse (assumed to be Gaussian) is double the average intensity $I_{p}$. The absorbed energy is defined by the absorbance $A$ and the absorbed energy density $\left[\mathrm{J} / \mathrm{cm}^{3}\right]$ at the end of the laser pulse is $W_{a}=A F_{p} / l_{a b}$, where the fluence $F_{p}=\int_{0}^{t_{p}} I_{p}(t) d t$. The electron density $N_{e}$ at the end of the pulse is defined by $\int_{0}^{t_{p}} I_{p}(t) d t$. The average intensity of the laser pulse is $I_{a}=c \varepsilon_{0} E_{0}^{2} / 2 \equiv c B_{0}^{2} /\left(2 \mu_{0}\right) \equiv E_{0} B_{0} /\left(2 \mu_{0}\right)$, where $E_{0}$ is the amplitude of the electric field strength $[\mathrm{V} / \mathrm{m}], B_{0}=E_{0} / c$ is the maximum magnetic field strength $[\mathrm{T}], \varepsilon_{0}, \mu_{0}$ are the permittivity and permeability of the free space, respectively. The generation of electrons by laser pules changes in time with the instantaneous intensity $I \propto E B$.

The energy change during one collision is $\Delta \epsilon_{e}=\frac{2 \epsilon_{o s c} \omega^{2}}{\nu^{2}+\omega^{2}}$, defined via the effective electron collision frequency and electron quiver energy $\epsilon_{o s c}=\frac{e^{2} E^{2}}{4 m_{e} \omega^{2}}$, where electron mass and charge are $m_{e}$ and $e$, respectively. When both the electron energy $\epsilon_{e}=\frac{A}{l_{a b s} N_{e}} \int_{0}^{t_{p}} I_{p} d t$, and $\Delta \epsilon_{e} \approx \epsilon_{\text {osc }}$ (the energy gained per electron in a single collision is comparable to that of the oscillation) are larger than the photon energy, then $\gamma \equiv \epsilon_{e} \Delta \epsilon_{e} /(\hbar \omega)^{2}>1$ and classical multi-photon absorption determines the ionisation rate - the so-called large frequency and moderate (low) field condition. When $\gamma \ll 1$, the tunneling ionisation becomes dominant (quantum case), i.e., low frequency, high-field conditions. At the intensity of $13 \mathrm{TW} / \mathrm{cm}^{2}$, which is typical for the breakdown threshold in silica and sapphire, $\gamma=2$. Avalanche ionisation, which is present at typical $\sim \mathrm{TW} / \mathrm{cm}^{2}$ irradiance at ambient conditions (especially at the longer near-IR wavelengths) is the dominant factor due to the avalanche rate scaling as $\lambda^{2}$.

To estimate the electron density at the end of a high-intensity ultra-short laser pulse $1-10 \mathrm{TW} / \mathrm{cm}^{2}$ and up to tens-of- TW $/ \mathrm{cm}^{2}$ reaching dielectric breakdown of a dielectric host (at $\operatorname{Re}(\varepsilon)=0$ ) - the multi-photon ionisation and impact (or avalanche) rates of electron generation $w_{m p i}$ and $w_{i m p}$, respectively, can be calculated by ${ }^{5}$ :

$$
\begin{aligned}
& w_{m p i} \approx \omega n_{p h}^{3 / 2}\left(\frac{\epsilon_{o s c}}{2 J_{i}}\right)^{n_{p h}}, \\
& w_{i m p} \approx \frac{\epsilon_{o s c}}{J_{i}}\left(\frac{2 \omega^{2} \nu}{\omega^{2}+\nu^{2}}\right),
\end{aligned}
$$



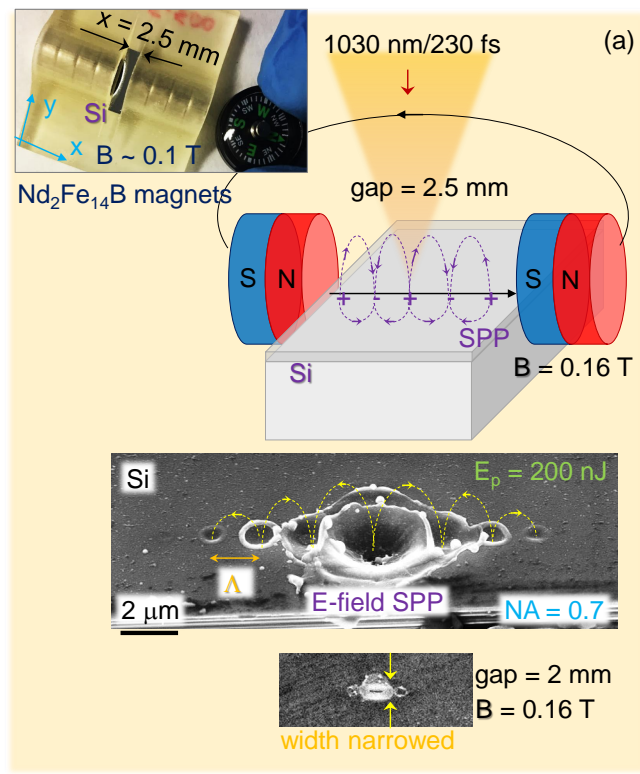

(a)

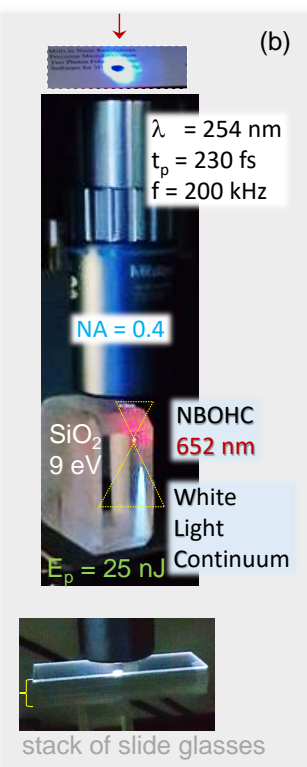

(b)

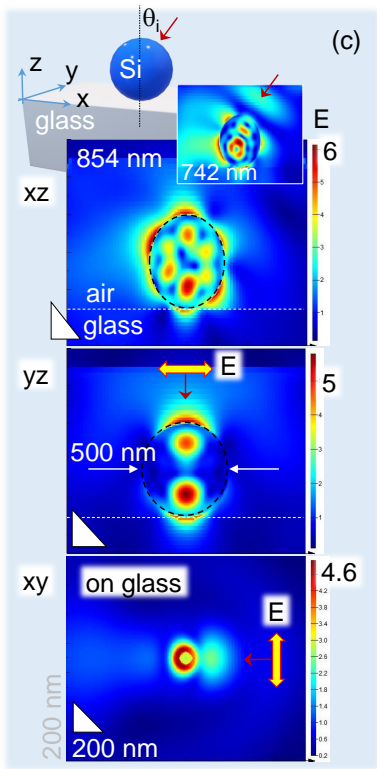

FIG. 6. On-surface and in-volume nanoscale modifications/printing. (a) Single pulse ablation pit at the focal spot of $1.22 \lambda / N A=1.8 \mu \mathrm{m}$ diameter with SPP-type imprint $\Lambda \sim 1 \mu \mathrm{m}$ along magnetic B-field lines; E-field lines of the SPP mode are shown by dashed-lines. Light intensity was $\sim 43 \mathrm{TW} / \mathrm{cm}^{2} /$ pulse. (b) Bond breaking in fused silica $\mathrm{SiO}_{2}$ detectable by luminescence of non-bridging oxygen hole center (NBHC) Si-O (bond breaking Si-O $\cdot \mathrm{Si}$ ); intensity $I_{p} \approx 23.1 \mathrm{TW} / \mathrm{cm}^{2} /$ pulse. Threshold of observable luminescence was $0.2 \mathrm{~nJ} /$ pulse or $0.18 \mathrm{TW} / \mathrm{cm}^{2}$. (c) FDTD calculated E-field enhancement at different cross sections of a Si-sphere of $0.5 \mu \mathrm{m}$ diameter of glass under a slanted $\theta_{i}=30^{\circ}$ incidence. See text for details.

where $n_{p h}=J_{i} / \hbar \omega$ is the number of photons necessary for atom ionisation.

These rate estimates are valid for $N_{e}$ excitation in the ENZ range (ENZ is $\left.\operatorname{Re}(\varepsilon)=0\right)$ and generation of electrons progress $\mathrm{as}^{5}$ :

$$
\frac{d N_{e}}{d t}=N_{e} w_{i m p}+N_{a} w_{m p i}
$$

which has a solution with an initial electron density $N_{0}$ :

$$
N_{e}=\left(N_{0}+\frac{N_{a} w_{m p i}}{w_{i m p}}\left[1-e^{-w_{i m p} t}\right]\right) e^{w_{i m p} t} .
$$

Here we reflect on the above formulae which provides the possibility of estimating electron density $N_{e}$ at any time across the lifetime of the laser pulse. The $N_{e}$ depends on the pulse intensity $I_{p}$ and is linked to the electron collision rates in plasma $\nu$, which tends to saturate as it approaches that of laser the frequency $\nu \approx \omega$. Due to shallow energy deposition into skin depth $l_{a b} \leq 100 \mathrm{~nm} \ll \lambda$, an interface between the host dielectric and metallic plasma state of Die-Met is created during the laser pulse. Such an interface can support the SPP mode which has wavelength determined from the maximum of $\operatorname{Re}(\beta)=2 \pi / \lambda_{s p}$ which is a standing wave $\left(v_{g} \rightarrow 0\right)$. Figure $3(\mathrm{~b})$ shows that at $N_{c r}$, when Die-Met transition into the metallic state $\varepsilon<1$ takes place, the dispersion dependencies $\omega$ vs. $\operatorname{Re}(\beta)$ and $\omega$ vs. $\operatorname{Im}(\beta)$ for surface bound propagating SPP and non-propagating modes, respectively, resembles dispersion $D(\omega)$ and absorption $A(\omega)$ lineshapes of the Lorentzian oscillator. The most efficient electron generation (large $A$ ) is at $N_{c r} / 2$ and most shallow energy deposition $l_{a n}<100 \mathrm{~nm}$ is at $2 N_{c r}$. This takes place at a small portion of the entire sub-1 ps pulse with an optical cycle $\sim 3$ fs.

The discussion in this section was focused on dielectrics and further developed for metals, with the main difference being that the ablation threshold occurs when the absorbed energy 
is in excess of the binding energy and electron work function $\left(\epsilon_{B}+\phi_{e s c}\right)^{5}$. The analysis presented was applied to the discussion of experimentally observed small-period ripple (LIPSS) formation in GaN as a SPP mode imprint on the plasma-GaN interface under irradiation from the air side ${ }^{10}$. The ripple period is taken as $\Lambda=\lambda_{s p} / 2$ and is presented as a standing wave imprint or formation of two counter-propagating SPPs forming a stationary interference field with period $\Lambda=\lambda_{s p} /\left(2 \sin \frac{\theta}{2}\right)=\lambda_{s p} / 2$ for the mutual angle between two SPP waves $\theta=\pi$. The orientation and direction of SPPs are defined by the polarisation of the incident E-field (only TM mode can launch the $\mathrm{SPP}^{11}$ ) and energy-axis mirror symmetry presented by the light-cone in Fig. 1.

Figure 6(a) shows a laser ablated pit made with a single $1030 \mathrm{~nm} / 230 \mathrm{fs}$ pulse, focused into a $1.8 \mu \mathrm{m}$ diameter area with the sample placed inside magnetic B-field ${ }^{12}$. The signature of the SPP-affected Si-air surface is evident and up to 10 times larger than the original energy deposition area. SEM images revealed the period of the pattern as $\Lambda \approx \lambda_{s p} / 2 \sim 1 \mu \mathrm{m}$ aligned along B-field. In a stronger B-field (smaller gap $x$ in (a)), ablation structures become elongated and narrowed as would be expected from a smaller cyclotron radiuselectrons move along magnetic field lines in a spinning trajectory of radius $r_{c}=\frac{m v}{q B}$, where $q$ is the charge $v$ the velocity.

Another example where plasmonic formation character is discussed is the 3D patterning of sub-wavelength $(\lambda / 20-\lambda / 4)$ structures of period $\Lambda=20-200 \mathrm{~nm}$ inside the volume of glass or crystal under multi-pulse exposure. Formation of the plasma region (required for the Die-Met change in permittivity) in the tightly focused laser pulse starts from direct bond breakage upon electronic excitation. In wide bandgap materials such as fused silica $E_{g} \approx 9 \mathrm{eV}$, cleaving of $\mathrm{Si}-\mathrm{O}-\mathrm{Si}$ bonds and creation of non-bridging oxygen hole centres (NBOHC) Si-O ${ }^{13}$ is visualised by the emission of $1.9 \mathrm{eV}$ red light. This occurs in the focal volume of the fs-laser pulse at the wavelength corresponding to $E_{g} / 2$, when two-photon absorption of the silica host is induced (Fig. 6(b)). Nano-gratings perpendicular to the linear polarisation of the incident light are formed in the focal region and can be patterned via beam/sample scanning over the required region with $3 \mathrm{D}$ direct write flexibility (similar as ripples on Si surface shown in Fig. 5).

For both surface or bulk irradiation at ENZ conditions (Fig. 6(a,b)), coupling of the laser pulse at normal incidence into SPP-mode requires phase matching (Fig. 1), which is relaxed when redirection/scattering light source becomes small, sub-wavelength. For example a nano-particle can act as a dipole and redirect light into the surface wave SPP (plasmonic response of a nanoparticle is discussed in the next Sec. IV). Figure 6(c) shows light field re-distribution and enhancement of a 1- $\mu$ m-diameter Si sphere on glass $(n=1.5)$ under a linearly polarised wave at angle of incidence $\theta_{i}=30^{\circ}$. Central cross sections and intensity maps at the glass interface reveals light localisation to tens-of-nm and its redirection, including reflection. This illustration shows that nanoscale localisation of light is expected for objects with size comparable to the wavelength and focal volumes of tightly focused laser pulses with lateral cross section $\sim \lambda / N A$ (a diffraction limited focal spot). Considering the resonant nature of such scattering, it is strongly dependent on angle of incidence and the wavelength linked to the permitivities in the interaction region. Again, it is an illustration that permittivity guides the light-matter interaction, which is constant theme of this chapter. The permittivity considered here (Eqn. 3) can also include Kerr nonlinearity by setting $\varepsilon_{n l}=\varepsilon_{K} I$ with $\varepsilon_{K}=2 \sqrt{\varepsilon_{i}} n_{2}\left[\mathrm{~cm}^{2} / \mathrm{W}\right]^{3}$.

\section{NANOPARTICLES IN HIGH INTENSITY LASER FIELD}

Next, light interaction with true nanoscale sized particles $\leq 100 \mathrm{~nm}$ is discussed. The fast evolving light energy deposition, from defect generation (Fig. 6(b)) to dielectric breakdown $\operatorname{Re}(\varepsilon)=0$, occurs during only a fraction of the ultra-short laser pulse $t_{p} \approx 300 \mathrm{fs}$. It is instructive to analyse light interaction with plasmonic (metallic or plasma) nano-volume; the collective effects of light scattering by nanoparticles at the ripple LIPSS formation conditions are actively discussed. 
nano-printing

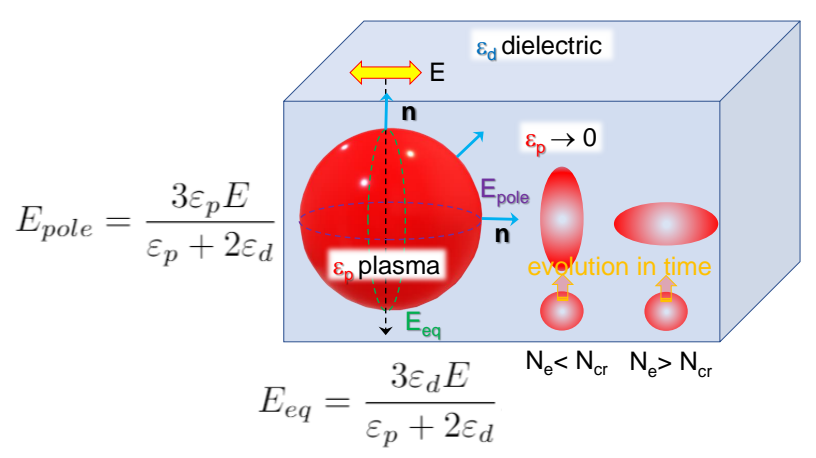

FIG. 7. Schematics of the local fields $E_{e q}$ and $E_{\text {pole }}$ near plasma nano-sphere dependent on $\varepsilon_{r}$, according to Eq. 16. Depending on the plasma density, the local field will be larger at equator or poles driving ionisation of host material at the interface.

Analytical formulas describing the local field distribution around plasma nano-spheres are derived following tutorial ref. ${ }^{14}$ with the notations given in Fig. 7 . It was previously discussed $^{15}$, that a plasma nano-sphere can be created in a sub-surface volume under irradiaiton by high-intensity laser pulses and be related to ripples $=$ formation ${ }^{16}$.

As a model, lets us consider plasma-like nano-spheres $\sim 20 \mathrm{~nm}$ which are surrounded by a dielectric environment with their size being much smaller compared to the laser wavelength $(\lambda \sim 1 \mu \mathrm{m})$, so electrostatic theory can be used to determine local field distribution around the plasma nano-sphere (Fig. 7). This field distribution drives evolution of ionisation and plasma formation upon absorption of the incident laser pulse at the plasma-host interface.

Electrical potential $\varphi_{\text {out }}$ outside the dielectric sphere with radius $R$ in the presence of uniform external electric field $E$ can be expressed as ${ }^{14}$ :

$$
\varphi_{\text {out }}=-E r \cos \Theta+\frac{\varepsilon_{r}-1}{\varepsilon_{r}+2} \frac{E R^{3}}{r^{2}} \cos \Theta,
$$

where $\Theta$ is the angle between external electric field $E$ and normal of the sphere surface $\mathbf{n}$ and $\varepsilon_{r}=\frac{\varepsilon_{p}}{\varepsilon_{d}}$ is relative permittivity expressed as ratio between plasma's $\varepsilon_{p}$ and dielectric $\varepsilon_{d}$ surrounding. The $\varphi_{\text {out }}$ potential describes a superposition of the applied E-field (by light) and that of a dipole located at the particle's centre. The formula for the corresponding local electric field outside the sphere $E_{l o c}$ in polar coordinates (unit vectors $\mathbf{e}_{\mathbf{r}}, \mathbf{e}_{\theta}$ ) is calculated using $E_{l o c}=-\nabla \varphi_{\text {out }}$ equation, which results in:

$$
E_{l o c}=\frac{3 E}{\varepsilon_{r}+2}\left(\varepsilon_{r} \cos \Theta e_{r}-\sin \Theta e_{\theta}\right) .
$$

The local field outside the plasma sphere near the equator, $E_{e q}$ (at $\Theta=\pi / 2$ ), and poles, $E_{\text {pol }}(\Theta=0, \pi)$ is respectively:

$$
E_{\text {eq }}=\frac{3 E}{\varepsilon_{r}+2}, \quad E_{\text {pole }}=\frac{3 \varepsilon_{r} E}{\varepsilon_{r}+2},
$$

or using $\varepsilon_{d}$ and $\varepsilon_{p}$ takes form:

$$
E_{e q}=\frac{3 \varepsilon_{d} E}{\varepsilon_{p}+2 \varepsilon_{d}}, \quad E_{\text {pole }}=\frac{3 \varepsilon_{p} E}{\varepsilon_{p}+2 \varepsilon_{d}} .
$$

The absolute value of Eq. 12 is given by:

$$
E_{l o c}=\frac{3 E}{\varepsilon_{r}+2} \sqrt{1+\left(\varepsilon_{r}^{2}-1\right) \cos ^{2} \Theta},
$$



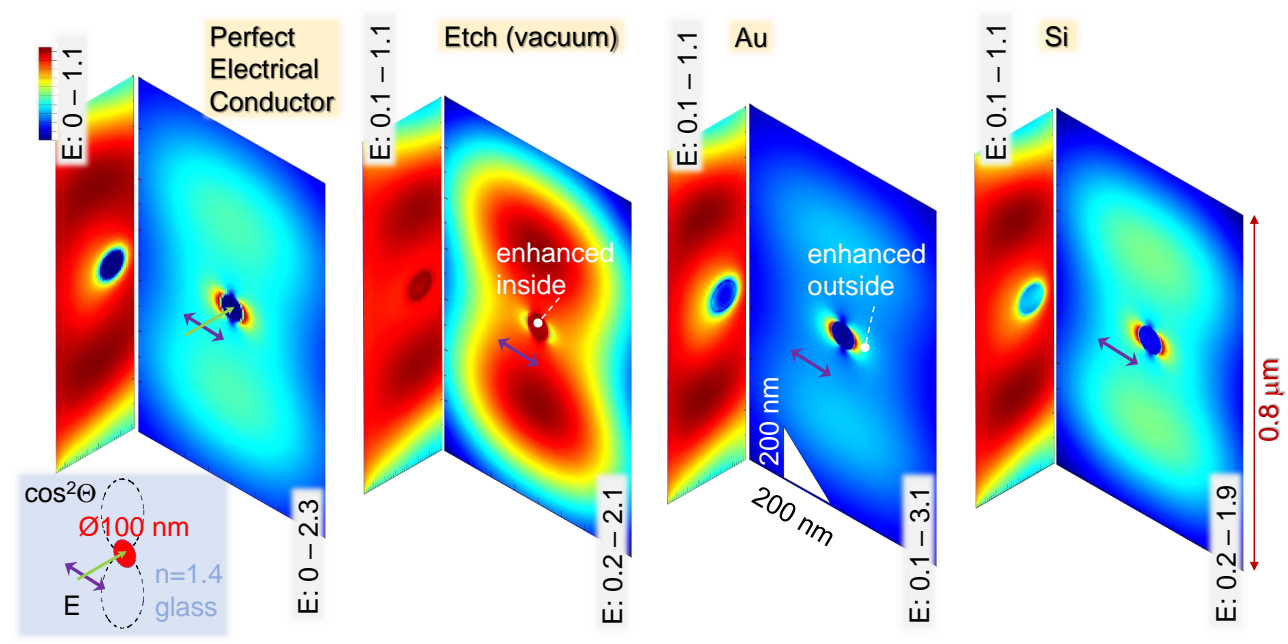

FIG. 8. FDTD simulation of light field distribution around 100-nm-diameter nanoparticles of different material inside silica glass $(n=1.4)$. Incident plane wave of linearly polarised light of wavelength $925 \mathrm{~nm}$; E-field of the incident light $\left|E_{0}\right|=1$, Si and Au properties are from the Lumerical database. Dipole scattering nature is recognisable as a $\cos ^{2} \theta$ intesnity profile; the vertical extension of the image is $\sim \lambda$.

or using $\varepsilon_{d}$ and $\varepsilon_{p}$ :

$$
E_{l o c}=3 E \frac{\sqrt{\varepsilon_{d}^{2}-\left(\varepsilon_{d}^{2}-\varepsilon_{p}^{2}\right) \cos ^{2} \Theta}}{\varepsilon_{p}+2 \varepsilon_{d}} .
$$

The local field enhancement factor, $D$, defined as the ratio between fields near equator and poles, is equal to the ratio between dielectric material and plasma permittivity:

$$
D=\frac{E_{e q}}{E_{p o l}}=\frac{\varepsilon_{d}}{\varepsilon_{p}}=\frac{1}{\varepsilon_{r}},
$$

where $\varepsilon_{r}$ is a relative permittivity. For a metallic/plasma nano-volume in a dielectric host under critical plasma conditions $0<\varepsilon_{r}<1$ (or $0<\varepsilon_{p}<\varepsilon_{d}$ ). Under these conditions, the local E-field is stronger near the equator compared to the poles, hence $D>1$ (Fig. 7). Plasma permittivity depends on electron density $\varepsilon_{p}=1-N_{e} / N_{c r}$ and is decreasing upon photo-excitation. As $N_{e}$ increases and plasma permittivity $\varepsilon_{p}$ decreases it reaches 0 at breakdown. At this condition, Eq. 17 predicts that field enhancement, $D$, is at a maximum. If critical electron density is further increased, $D$ decreases and at $\varepsilon_{r}<-1$ field becomes stronger near poles and plasma growth direction changes from normal to parallel to the surface (Fig. 7). The local field can also be presented via the enhancement factor $L=\frac{3}{\varepsilon_{r}+2}$, which is explicitly presented via permittivities in Eqn. 14. The local field enhancement is directly related to the surface enhanced Raman scattering (SERS).

It is noteworthy that the local field $E_{e q}$ or $E_{\text {pole }}$ is growing at the $-\left|\varepsilon_{p}\right|=2 \varepsilon_{d}$, the so-called Frölich condition when the denominator in Eqn. 14 approaches zero. The local E-field bound to the interfaces of micro-volumes can reach large enhancements of $10-50$ times and localisation down to tens-of-nm as shown by FDTD, which is exact the solution of Maxwell's equations (Figs. 4, 6(c)).

Light scattering from single $100 \mathrm{~nm}$ diameter nano-spheres of different materials are shown in Fig. 7 calculated by FDTD (Lumerical). The pattern of dipole scattering emerges around the laser-driven plasmon, localised on the nano-sphere, i.e. light is not emitted along the dipole axis (E-polarisation) and has toroidal a distribution perpendicular to the dipole. A perfect electrical conductor represents material with $E=0$ within it and has 
nano-printing

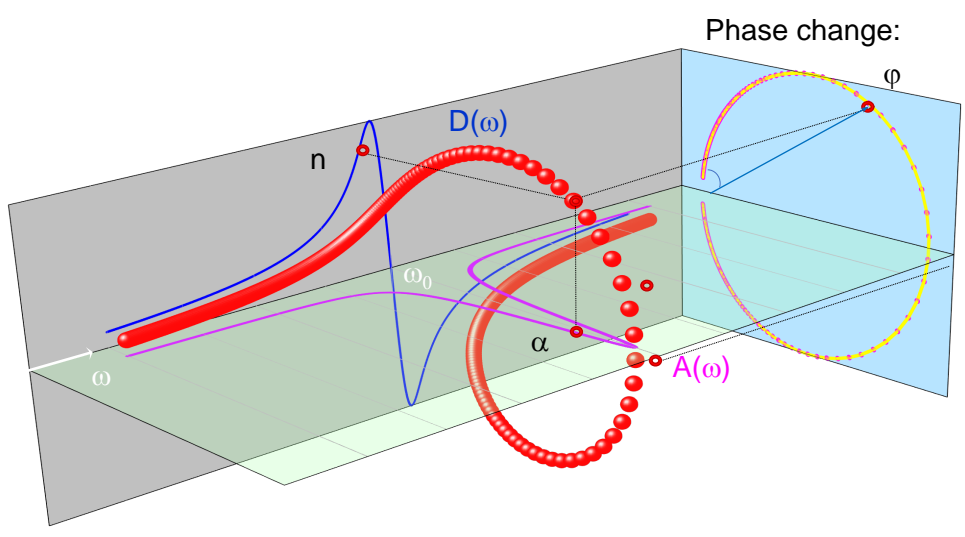

FIG. 9. Absorption $A(\omega)=\tau /\left(1+\left(\omega-\omega_{0}\right)^{2} \tau^{2}\right)$ and dispersion $D(\omega)=\left(\omega-\omega_{0}\right) \tau^{2} /\left(1+\left(\omega-\omega_{0}\right)^{2} \tau^{2}\right)$ lineshapes for the Lorentzian oscillator near resonance $\omega_{0}^{8} ; \tau$ is the relaxation time. Real part of the refractive index $n$ and imaginary part $\kappa$ are inter-related; absorption coefficient $\alpha=4 \pi \kappa / \lambda\left[\mathrm{cm}^{-1}\right]$.

$100 \%$ reflection and $0 \%$ absorption. The empty space (etch) sample simulates an empty nano-sphere inside glass host. At $\lambda=925 \mathrm{~nm}$ wavelength, all $100 \mathrm{~nm}$ spheres show the same geometry of light redistribution around it. However, the empty (etch) sample shows light intensity is enhanced inside it as compared to the glass side of the interface (similar case is discussed in the Sec. $\mathrm{V}$ for nano-hole on a surface). The calculated cross sections have extensions smaller than the wavelength of light and shows how such oscillating dipoles act for light redirection and can deliver the required phase matching (Fig. 1). Nanoparticles smaller that the wavelength of light have scattering $C_{s c}$ and absorption $C_{a}$ cross sections dependent on the polarizability $\alpha^{11}$ :

$$
C_{s c}=\frac{1}{6 \pi}\left(\frac{2 \pi}{\lambda}\right)^{4}|\alpha|^{2} ; \quad C_{a}=\frac{2 \pi}{\lambda} \operatorname{Im}(\alpha) ; \quad \alpha=3 V \frac{\varepsilon_{m}-\varepsilon_{d}}{\varepsilon_{m}+2 \varepsilon_{d}}
$$

where $V$ is the volume of nanoparticle with permittivity $\varepsilon_{m}$ in the dielectric host $\varepsilon_{d}$. The plasmon resonance localised on a nanoparticle is dependent on the particle size and shape. For spherical metallic nanoparticles, the Maxwell-Garnett (MG) medium theory defines the effective permittivity dependent on the volume fraction $F$ of nanoparticles as $\varepsilon_{M G}=$ $\varepsilon_{d} \frac{3+2 F \alpha^{\prime}}{3-F \alpha^{\prime}}$, where $\alpha^{\prime}$ is the constant proportional to the polarisability of nanoparticles ${ }^{17}$. The resonant plasmon absorption wavelength of the localised plasmon is then given as ${ }^{17}$ :

$$
\lambda_{3 D}=\lambda_{p} \sqrt{1+\left(\frac{F+2}{1-F}\right) n_{s}^{2}}
$$

where $n_{s}$ is the refractive index surrounding MG medium. This wavelength and the corresponding frequency can be considered as a localised 3D plasmon, while the SPP mode is the $2 \mathrm{D}$ plasmon and bulk plasmon with $\lambda_{p}$ ( $\omega_{p}$ frequency e.g., $\lambda_{p}=131 \mathrm{~nm}$ for $\mathrm{Au}$ ). In all those cases of 1D, 2D and 3D plasmonic resonances, the absorbance spectral lineshape can be considered as the Lorentzian where the real and imaginary parts of the refractive index (and permittivity) are inter-related as shown in the complex plane presentation (Fig. 9) to reveal phase evolution in the vicinity of resonance ${ }^{8}$. 


\section{NANOLITHOGRAPHY BY DIRECT WRITE WITH OPTICAL NEAR-FIELD}

The localisation and enhancement of light intensity within a hollow $100 \mathrm{~nm}$ diameter nano-void (vacuum case in Fig. 8) makes it possible to ionise the inner glass-vacuum interface. This is similar to the ionisation of the surrounding host by a metallic/plasma nanoparticle (Fig. 7; the interface from the outside of nanoparticle). It is exactly this principle of light localisation and enhancement inside a nano-hole $20-30 \mathrm{~nm}$ in diameter that was used as a "nano-pen", which inscribed nano-grooves of the similar $\sim 20-30 \mathrm{~nm}$ width throughout the entire thickness of a $\mathrm{TiO}_{2}$ film of $23 \mathrm{~nm}$ deposited on a glass substrate ${ }^{18}$. This direct write nanolithography (Fig. 10(a)) is realised as a "nano-pen", which is steered by linear polarisation of a fs-laser beam focused onto a $1.2 \mu \mathrm{m}$ focal spot $(N A=0.8$, $\lambda=0.8 \mu \mathrm{m}$ ) with a nano-film of titania acting as a "resist". Interestingly, nano-grooves can be written as close as width of the groove. The reason for such performance is revealed by the FDTD simulations in Fig. 10(b). A nano-hole (at the centre of the calculation field) of $30 \mathrm{~nm}$ diameter, along with grooves with orientations (vertical or horizontal), widths (20 or $30 \mathrm{~nm}$ ), and end shape (rounded or rectangular) were illuminated at $\lambda=794 \mathrm{~nm}$ by a plane wave (no focusing). Light field enhancement inside the nano-hole and nano-grooves depends on mutual orientation of linear polarisation (E-field) and the nano-void. For the nano-hole, the light intensity is the largest inside it and has a sudden drop just across the interface into titania along the E-field orientation. For nano-grooves, enhancement is expressed more when the groove is perpendicular to the polarisation. For grooves aligned to the E-field, there is almost no enhancement (barely recognisable in Fig. 10(b)).

In both nano-hole and nano-groove cases, the reason for light enhancement and its polarisation dependence is the boundary condition to the normal components for the displacement $D^{(n)} . D_{2}^{(n)}-D_{1}^{(n)}=\sigma$ for the interface between two materials titania (1) and air (2), where $\sigma$ is the surface charge density (unbound charges or free); another condition is continuity of the tangential components $E_{1}^{(t)}=E_{2}^{(t)}$. For the dielectrics discussed here $\sigma=0$ and
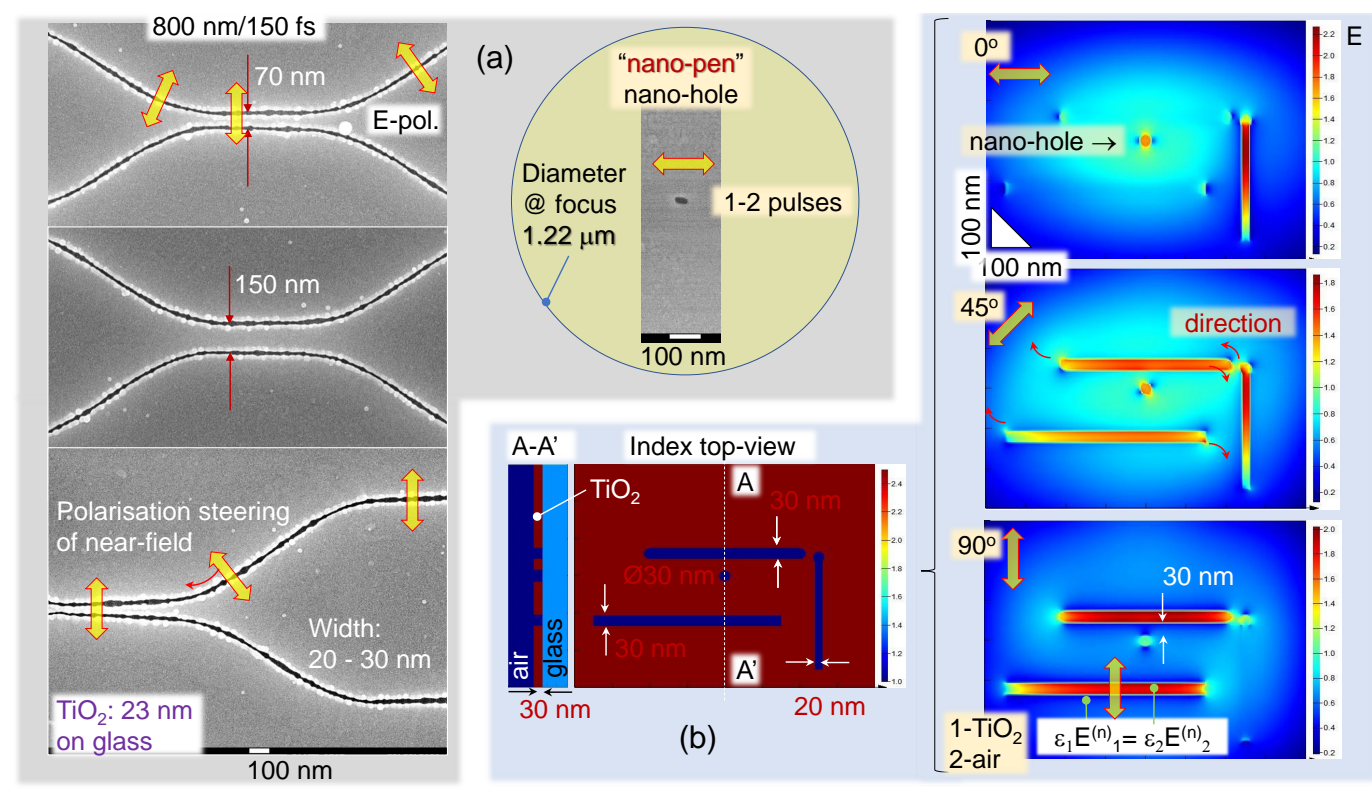

FIG. 10. Nano-hole - "nano-pen" ${ }^{18}$ : polarisation steering of optical near-field (experiment (a) and numerical model (b)). (a) SEM images of nano-grooves ablated by near-field enhanced inside a nano-hole, which was initiated by irradiation of 1-2 threshold intensity pulses. (b) FDTD light field enhancement simulations for nano-grooves and a nano-hole showing E-field enhancement dependent on polarisation (a cool-hot color scale); wavelength of light $\lambda=794 \mathrm{~nm}$ and refractive index of $\mathrm{TiO}_{2}$ and glass substrate were set to $n=2.5$ and 1.4 values, respectively (see text for details). 
$\varepsilon_{1} E_{1}^{(n)}=\varepsilon_{2} E_{2}^{(n)}$. This boundary condition is based on the E-field strength which is the product of $\mathbf{E} \cdot \mathbf{n}_{\mathbf{1 2}}=E \cos \theta$. With $\mathbf{n}_{\mathbf{1 2}}$ being the normal to the interface, it defines the steering capability of the optical near-field upon illumination (see arrow markers in $\theta=\pi / 4$ calculated light-field in Fig. 10(b)). The intensity $I \equiv E^{2}$ and the dipole pattern of intensity $\cos ^{2} \theta$ opens the possibility to redirect the maximum intensity at the interface by changing the linear polarisation orientation of the illumination. For the titania-air boundary $\left(n_{1}=2.5\right.$ vs. $\left.n_{2}=1\right)$, the light intensity inside the air gap (2) is enhanced with respect to the field inside titania (1) by $I_{2}=\left|E_{2}\right|^{2}=\left(\frac{n_{1}^{2}}{n_{2}^{2}}\right)^{2} E_{1}^{2} \approx 39 I_{1}$. This is immense enhancement in the near-field and is harnessed to ablate nano-grooves by direct laser writing (Fig. 10(a)). Figure 10(b) also reveals that close proximity ablation of nano-grooves does not cause light field enhancement between the nano-hole and nano-groove ( $\operatorname{see} \theta=\pi / 2$ panel). Conversely, a lower light field intensity occurs between them. The FDTD calculations are made for plane wave incidence, while the actual nano-ablation (a) was made with a focused fs-laser beam into $\mathrm{a} \sim 1.2 \mu \mathrm{m}$ focal spot (see circle in (a) overlaid with SEM image of nano-hole). When the laser pulse energy was changed by only $\sim 10 \%$, no ablation of pristine titania film took place. The enhancement in a nano-hole by more that $20 \%$ above or below the ablation threshold can be well controlled during writing. This experimental observation is well supported by the FDTD intensity distribution maps at the tips of grooves (sharp or rounded tips do not cause fundamental differences in steering and enhancement of the near-fields).

Direct fs-laser writing at the nanoscale using optical near-field enhancement, which is directed and steered by the polarisation orientation of the incoming beam, has the distinct advantage of avoiding proximity effects encountered in particle beam lithographies (electron and ion e/i) - where resist exposure by secondary electrons reduce resolution. Noteworthy, even though the single feature size in e/i-beam lithographies can be a few-nm, the resolution when two lines/dots have to be defined close together in the resist suffers from the proximity effects. In the as-described nano-pen lithography (branded as optical-focused ion beam (OFIB $)^{18}$ ), the proximity issue is resolved naturally and laser focal spot does not determine the resolution of nano-patterning. This approach is vacuum-less and can be carried out with indefinite write fields (no stitching required). There is a good practical tolerance of nanopen writing for the axial displacement of laser focus due to the focal spot having typical axial/lateral $\sim 3 \mu \mathrm{m} / 1 \mu \mathrm{m}$ dimensions. Hence, the technique is applicable to curved surfaces and works with dry-optics $(N A<1)$ required for fast beam/sample scanning. The demonstrated principle used a 23 -nm-thick titania film on glass ${ }^{18}$. This helped produce a large field enhancement $n^{4} \approx 40$ due to large refractive index $n=2.5$ of titania. However, the demonstrated principle of the enhancement is present even for a nano-hole on the surface of any material. It is best illustrated in the $3 \mathrm{D}$ void case in Fig. 8 where the nano-void is surrounded by the same host medium. A single nano-line was recorded using ablation on sapphire ${ }^{19}$ by starting with ablation of ripples and reducing the laser pulse energy to form a single nano-groove. It is noteworthy that there is no E-field enhancement expanding into substrate (Fig. 10(b)) or air (not shown here for brevity), which is a welcome feature. This is especially relevant for electronic materials which can be damaged with high energy $\sim 100 \mathrm{keV}$ electron beams used in high resolution e-beam lithography or material implantation as is the case for i-beam lithography ${ }^{20}$.

In order to guide nano-ablation to within the hole/groove, the working recipe for this optical direct write near-field nano-pen is: 1. make a seed nano-hole/void, 2 . use at slightly lower laser pulse energy during scanning/writing, 3. steer the ablation trace by polarisation of incoming laser beam. Polarisation steering was the same as for surface patterning (Fig. 5) control by rotating a $\lambda / 2$-waveplate along the scan trajectory. With increasing complexity and versatility of combined electron, ion, photon micro-fabrication tools $^{21}$, there are different methods for how to create a seed nano-hole to be used in nano-pen writing. Such a nano-hole is a structure which grows into ripples under multiple irradiation as shown in lithium niobate ${ }^{22}$.

Writing speed can be high and almost debris-free due to minute material removal. The velocity of air molecules at normal conditions is $v_{r m s}=\sqrt{3 k T / m}$, i.e. $480 \mathrm{~m} / \mathrm{s}$ for $\mathrm{O}_{2}$ and 
nano-printing

$510 \mathrm{~m} / \mathrm{s}$ for $\mathrm{N}_{2}$. During a laser pulse of $t_{p}=1 \mathrm{ps}, \mathrm{N}_{2}$ molecules only move $0.5 \mathrm{~nm}$. The free path of molecules $l_{f}=1 /\left(\sqrt{2} \pi n_{0} \sigma\right)=k T /(\sqrt{2} \pi p \sigma)$, where the $n_{0}$ is number density of molecules, $\sigma=a^{2}$ is the cross section of molecules defined by their size $a, p$ is the pressure, $k$ is the Boltzmann constant, and $T$ is the absolute temperature. The value of $l_{f} \approx 60 \mathrm{~nm}$ in air with $n_{0}=2.69 \times 10^{19} \mathrm{~cm}^{-3}$. During a sub-1 ps laser pulse pulse used to write $20 \mathrm{~nm}$ width lines, the movement of molecules in air is less than $0.5 \mathrm{~nm}$ under ambient conditions. The cross-sections for the light-matter interaction volume is smaller than $l_{f}^{3}$ and so there is an equivalence to close-to-vacuum conditions during laser fabrication during this nano-pen writing. Low molecular number density in the light-matter interaction volume is always advantageous for high precision nanofabrication and nanolithography.

\section{LOCALISATION OF ENERGY DEPOSITION}

The common feature of the plasmonic nature of nanoprinting is the $3 \mathrm{D}$ localisation of energy deposition into the skin depth $l_{a b}=\lambda /(4 \pi \kappa)$. How much light energy is absorbed is also dependent the on portion of reflected light $R=f(n, \kappa)$. In the case of nanolithography with a nano-pen, light localisation was delivered by an empty/void nanostructure. In all the 3D, 2D, and 1D cases discussed, optical near-field is the way for light concentration and localisation. We have shown that the volumetric energy density Energy $/$ Volume $\sim \mathrm{J} / \mathrm{mm}^{3}$ is the right measure for the additive mode of $3 \mathrm{D}^{(+)}$printing by ultra-short laser pulses ${ }^{23}$, e.g., in the case of polymerisation.

In the case of ablation $\left(3 \mathrm{D}^{-}\right.$fabrication), fine tuning of the optimum ablation rate is achieved by, changing the pulse number per irradiation spot, using beam scanning ${ }^{24}$, and control over the number of pulses per burst ${ }^{25}$. For comparison of different fabrication conditions, the volume $\left[\mathrm{mm}^{3}\right]$ ablated per $1 \mathrm{~W}$ average power per min: $V_{a} \sim \mathrm{mm}^{3} / \mathrm{W} / \mathrm{min}$ $\sim \mathrm{mm}^{3} /(\mathrm{W} . \mathrm{s}) \sim \mathrm{mm}^{3} / \mathrm{J}$ is used. This is the ablated volume-per-energy delivered by the laser for subtractive machining $\left(3 \mathrm{D}^{(-)}\right.$printing). It is not surprising that accounting for the energy deposition in the light-matter interaction volume is the essential measure for both the additive and subtractive $3 \mathrm{D}^{(+)}$and $3 \mathrm{D}^{(-)}$modes of $3 \mathrm{D}$ fabrication.

New modes of laser operation brings the capability of combining ultra-short pulses into $\mathrm{MHz}-\mathrm{GHz}$ bursts with a controlled number of pulses per burst ${ }^{25}$. It was shown that this burst mode of operation delivers ablation rates for metal and dental tissue on the order of $3 \mathrm{~mm}^{3} / \mathrm{min}$. This is the rate that reaches that of current Electrical Discharge Machining/Grinding (EDM/G) computer numerical control (CNC) tools. This parity between material removal rate by discharge spark and laser beam was achieved in 2016 . The burst mode advantage is the ability to fine tune material removal to the most efficient fluence $\left[\mathrm{J} / \mathrm{cm}^{2}\right]^{26}$, which was empirically determined to be $e^{2}=7.4$ times larger than the ablation threshold for the given material ${ }^{27}$. This scaling is consistent with optical energy delivery into the skin depth, rather than ablation via heating of the irradiated and surrounding volume, which is the case for longer pulses.

Among other examples of plasmonic modification of materials are photo-reduction of $\mathrm{Ag}$ in a polymer and SPP imprint ${ }^{28}$, localised plasmon induced polymerisation in nanogaps $^{29,30}$, polymerisation enhancement at the tips of black-Si ${ }^{31}$.

\section{CONCLUSIONS AND OUTLOOK}

Since the year 2000, the average laser power of ultra-short (sub-1 ps) pulsed lasers has increased as Power $=2^{N / 2}$ with $N$ - number of the years from beginning of the trend, which parallels Moore's law for number of transistors in an integrated circuit (based on Amplitude report of ultra-short laser product line $\mathrm{e}^{32}$ ). Parallels can be drawn between photons and transistors, with laser units reducing in size while increasing the number of photons in time (intensity in number of photons per time $N \times \hbar \omega / t_{p}[\mathrm{~W}]$ ). Miniaturisation has been the driving force of Moore's law for last 50 years in microelectronics. One can expect the same 
nano-printing

impact for ultra-short pulsed lasers, increasing and expanding laser processing for high speed and large surface areas (or volume modification). Ultra-short laser pulses can control energy deposition both in time and space down to the nanoscale, driving local modification via melting, evaporation, and ablation. With this flexibilty, applications are expected to grow.

\section{ACKNOWLEDGMENTS}

This work was supported by the ARC Discovery DP190103284 and Linkage LP190100505 projects. SJ is grateful to Workshop-of-Photonics for the technology transfer project and fs-laser industrial 3D printing setup based on Pharos laser.

${ }^{1}$ R. L. Olmon, B. Slovick, T. W. Johnson, D. Shelton, S.-H. Oh, G. D. Boreman, and M. B. Raschke, "Optical dielectric function of gold," Physical Review B 86, 235147 (2012).

${ }^{2}$ E. G. Gamaly and A. V. Rode, "Ultrafast re-structuring of the electronic landscape of transparent dielectrics: new material states (Die-Met)," Applied Physics A 124, 278 (2018).

${ }^{3}$ E. G. Gamaly and A. V. Rode, "Transient optical properties of dielectrics and semiconductors excited by an ultrashort laser pulse," J. Opt. Soc. Am. B 31, C36 (2014).

${ }^{4}$ B. Farid and R. W. Godby, "Cohesive energies of crystals," Physical Review B 43, 14248-14250 (1991).

${ }^{5}$ E. G. Gamaly, A. V. Rode, B. Luther-Davies, and V. T. Tikhonchuk, "Ablation of solids by femtosecond lasers: Ablation mechanism and ablation thresholds for metals and dielectrics," Phys. Plasmas 9, 949 (1991).

${ }^{6}$ S. Juodkazis, K. Nishimura, S. Tanaka, H. Misawa, E. E. Gamaly, B. Luther-Davies, L. Hallo, P. Nicolai, and V. Tikhonchuk, "Laser-induced microexplosion confined in the bulk of a sapphire crystal: Evidence of multimegabar pressures," Phys. Rev. Lett. 96, 166101 (2006).

${ }^{7}$ E. E. Gamaly, S. Juodkazis, K. Nishimura, H. Misawa, B. Luther-Davies, L. Hallo, P. Nicolai, and V. Tikhonchuk, "Laser-matter interaction in a bulk of a transparent solid: confined micro-explosion and void formation," Phys. Rev. B 73, 214101 (2006).

${ }^{8}$ A. G. Marshall, "Dispersion vs. absorption (DISPA): A magic circle for spectroscopic line shape analysis," Chemometrics \& Intelligent Laboratory Systems 3, 261-275 (1988).

${ }^{9}$ L. Wang, Q.-D. Chen, X.-W. Cao, R. Buividas, X. Wang, S. Juodkazis, and H.-B. Sun, "Plasmonic nano-printing: large-area nanoscale energy deposition for efficient surface texturing," Light: Sci. Appl. 6, e17112 (2017).

${ }^{10}$ K. Miyazaki and G. Miyaji, "Nanograting formation through surface plasmon fields induced by femtosecond laser pulses," J. Appl. Phys. 114, 153108 (2013).

${ }^{11}$ S. A. Maier, "Plasmonics: fundamentals and applications," (Springer, 2007).

${ }^{12}$ J. Maksimovic, S. Ng, T. Katkus, B. Cowie, and S. Juodkazis, "External field-controlled ablation: Magnetic field," Nanomaterials 9, 1662 (2019).

${ }^{13}$ S. S. Mao, F. Qu(e)r(e), S. Guizard, X. Mao, R. E. Russo, G. Petite, and P. Martin, "Dynamics of femtosecond laser interactions with dielectrics," Appl. Phys. A 79, 1695-1709 (2004).

${ }^{14}$ L. Grechko, V. Sugakov, O. Tomasevich, and A. Fedorchenko, "Sbornik zadach po teoreticheskoj fizike," (Vysshajashkola, Moskva (in Russian), 1972).

${ }^{15}$ R.Buividas, L. Rosa, R. Šliupas, T. Kudrius, G. Šlekys, V. Datsyuk, and S. Juodkazis, "Mechanism of fine ripple formation on surfaces of (semi)transparent materials via a half-wavelength cavity feedback," Nanotechnology 22, 055304 (2011).

${ }^{16}$ R. Buividas, M. Mikutis, and S. Juodkazis, "External field-controlled ablation: Magnetic field," Prog. Quantum Electron. 38, 119-156 (2014).

${ }^{17}$ V. N. Rai, A. K. Srivastava, C. Mukherjee, and S. K. Deb, "Surface enhanced absorption and transmission from dye coated gold nanoparticles in thin films," Appl. Optics 51, 2606-2015 (2012).

${ }^{18}$ Z.-Z. Li, L. Wang, H. Fan, Y.-H. Yu, Q.-D. Chen, S. Juodkazis, and H.-B. Sun, "O-FIB: far-field-induced near-field breakdown for direct nanowriting in an atmospheric environment," Light: Sci. Appl. 9, 41 (2020).

${ }^{19}$ R. Buividas, S. Rekštytè, M. Malinauskas, and S. Juodkazis, "Nano-groove and 3D fabrication by controlled avalanche using femtosecond laser pulses," Opt. Mat. Express 3, 1674-1686 (2013).

${ }^{20}$ G. Seniutinas, A. Balčytis, I. Rèklaitis, F. Chen, J. Davis, C. David, and S. Juodkazis, "Tipping solutions: emerging 3D nano-fabrication/-imaging technologies," Nanophotonics 6, 923-941 (2017).

${ }^{21}$ M. P. Echlin, M. Straw, S. Randolph, J. Filevich, and T. M. Pollock, "The tribeam system: Femtosecond laser ablation in situ SEM," Materials Characterization 100, 1-12 (2015).

${ }^{22}$ H. Shimizu, G. Obara, M. Terakawa, E. Mazur, and M. Obara, "Evolution of femtosecond laser-induced surface ripples on lithium niobate crystal surfaces," Appl. Phys. Express 6, 112701 (2013).

${ }^{23}$ E. Skliutas, M. Lebedevaite, E. Kabouraki, T. Baldacchini, J. Ostrauskaite, M. Vamvakaki, M. Farsari, S. Juodkazis, and M. Malinauskas, "Polymerization mechanisms initiated by spatio-temporally confined light," Nanophotonics 10, 1211-1242 (2021). 
nano-printing

${ }^{24}$ E. Vanagas, J. Kawai, D. Tuzilin, I. Kudryashov, A. Mizuyama, K. G. Nakamura, K.-I. Kondo, S.Y. Koshihara, M. Takesada, K. Matsuda, S. Juodkazis, V. Jarutis, S. Matsuo, and H. Misawa, "Glass cutting by femtosecond pulsed irradiation," J. Microlith. Microfab. Microsyst. 3, 358-363 (2004).

${ }^{25}$ C. Kerse, H. Kalaycioğlu, P. Elahi, B. Çetin, D. Kesim, O. Akçaalan, S. Yavaş, M. Aşık, B. Öktem, H. Hoogland, R. Holzwarth, and F. Ilday, "Ablation-cooled material removal with ultrafast bursts of pulses," Nature 537, 84-88 (2016).

${ }^{26}$ D. Förster, B. Jäggi, A. Michalowski, and B. Neuenschwander, "Review on experimental and theoretical investigations of ultra-short pulsed laser ablation of metals with burst pulses," Materials 14, 3331 (2021).

${ }^{27}$ N. Hodgson, A. Steinkopff, S. Heming, H. Allegre, H. Haloui, T. Lee, M. Laha, and J. VanNunen, "Ultrafast laser machining: process optimization and applications," in SPIE Laser Applications in Microelectronic and Optoelectronic Manufacturing (LAMOM) XXVI, Vol. 11673 (SPIE, Bellingham, Washington, USA, 2021) pp. 11673-08.

${ }^{28}$ Y.-K. Sun, L. Wang, M. Kamano, and S. Juodkazis, "Plasmonic nano-imprinting by photo-doping," Opt. Lett. 43, 3786-3789 (2018).

${ }^{29}$ K. Ueno, S. Juodkazis, T. Shibuya, Y. Yokota, V. Mizeikis, K. Sasaki, and H. Misawa, "Nanoparticle plasmon-assisted two-photon photolymerization induced by incoherent excitation source," J. Am. Chem. Soc. 130, 6928-6929 (2008).

${ }^{30}$ K. Ueno, S. Juodkazis, T. Shibuya, V. Mizeikis, Y. Yokota, and H. Misawa, "Nano-particle-enhanced photo-polymerization," J. Phys. Chem. C 113, 11720-11724 (2009).

${ }^{31}$ A. Žukauskas, M. Malinauskas, A. Kadys, G. Gervinskas, G. Seniutinas, S. Kandasamy, and S. Juodkazis, "Black silicon: substrate for laser 3D micro/nano-polymerization," Optics Express 21, 6901-6909 (2013).

${ }^{32}$ E. Mottay, "Femtosecond lasers for high throughput surface engineering," in SPIE Photonics West Industry, Vol. 11768 (SPIE, Bellingham, Washington, USA, 2021) pp. 11768-07. 